\title{
Tuning the Band Gap in $\mathrm{CsPbBr}_{3}$ Through Sr Substitution
}

\author{
Daniel B. Straus* and R. J. Cava* \\ Department of Chemistry, Princeton University, Princeton, NJ 08544 USA \\ *Authors to whom correspondence should be addressed. Email: dstraus@princeton.edu, \\ rcava@princeton.edu
}

\begin{abstract}
The ability to continuously tune the band gap of a semiconductor allows its optical properties to be precisely tailored for specific applications. We demonstrate that the band gap of the halide perovskite $\mathrm{CsPbBr}_{3}$ can be continuously widened through homovalent substitution of $\mathrm{Sr}^{2+}$ for $\mathrm{Pb}^{2+}$ using solid-state synthesis, creating a material with the formula $\mathrm{CsPb}_{1-\mathrm{x}} \mathrm{Sr}_{\mathrm{x}} \mathrm{Br}_{3}(0 \leq \mathrm{x} \leq 1) . \mathrm{Sr}^{2+}$ and $\mathrm{Pb}^{2+}$ form a solid solution in $\mathrm{CsPb}_{1-}$ ${ }_{x} \mathrm{Sr}_{\mathrm{x}} \mathrm{Br}_{3}$. Pure $\mathrm{Cs} \mathrm{PbBr}_{3}$ has a band gap of 2.29(2) eV, which increases to 2.64(3) eV for $\mathrm{Cs} \mathrm{Pb}_{0.25} \mathrm{Sr}_{0.75} \mathrm{Br}_{3}$. Density-functional theory calculations support the hypothesis that $\mathrm{Sr}$ incorporation widens the band gap without introducing mid-gap defect states. These results demonstrate that homovalent B-site alloying is a viable method to tune the band gap of halide perovskites without introducing compensating defects that form when B-site cations of a different charge are introduced.
\end{abstract}

\section{TOC Graphic}

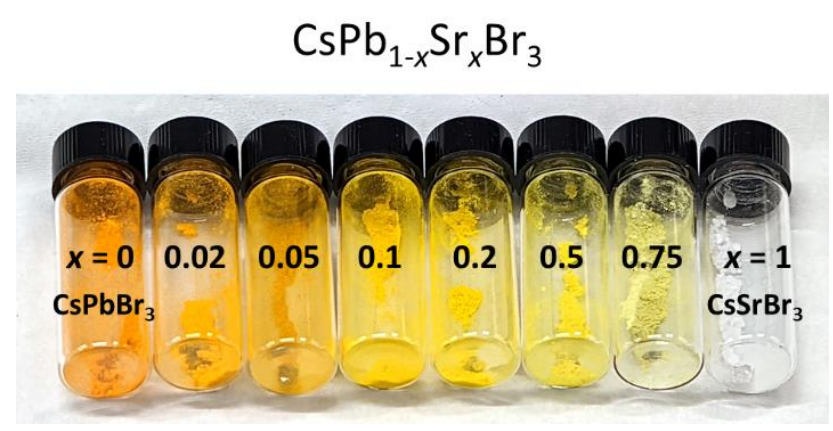


Halide perovskites are best known as semiconductors that have large absorption cross-sections and long carrier diffusion lengths, which allows them to serve as a high-performing active layer in solar cells. ${ }^{1-6}$ They typically have the chemical formula $\mathrm{ABX}_{3}$, where $\mathrm{A}$ is a $1+$ cation, $\mathrm{B}$ a $2+$ cation, and $\mathrm{X}$ a halogen. The relative sizes of the ions restricts the formation of halide perovskites, formalized through the Goldschmidt tolerance factor. ${ }^{7,8}$ The band gap of a material is generally determined by the relative electronegativities of its constituents and its electron count, and halide perovskites are no exception. ${ }^{9-11}$ Tunable band gaps are desirable because they allow the optical properties of a material to be optimized for a specific application, such as fabricating a light-emitting diode that emits a specific color of light. ${ }^{12}$ To tune the band gap of a halide perovskite, a frequently employed method is to change the halogen — chloridebased halide perovskites generally have wider band gaps than bromide-based perovskites, which have wider band gaps than iodide-based perovskites, for example. ${ }^{13}$ Incorporating a mixture of halogens allows the band gap to be continuously tuned. Unfortunately, under illumination, phase segregation of the halogens can occur, which lowers the effective band gap of a mixed halide perovskite material to that of one of the end-member perovskites. ${ }^{13}$ Incorporating mixtures of A-site cations also allows for limited band gap tunability, ${ }^{14}$ but this method is limited in application for all-inorganic lead and tin halide perovskites because $\mathrm{Cs}^{+}$is the only inorganic cation large enough to stabilize a pure-phase perovskite structure. ${ }^{15,16}$

Another method to tune the band gap is by replacing a fraction of the B-site cations with an alternate cation. This can be accomplished by creating an ordered double perovskite ${ }^{17}$ with the formula $\mathrm{A}_{2} \mathrm{BB}^{\prime} \mathrm{X}_{6}$ where $\mathrm{B}$ is a $1+$ and $\mathrm{B}$ ' a $3+$ cation, maintaining an average $2+$ charge for the $\mathrm{B}$ site. ${ }^{11,18,19}$ This is also found for vacancy-ordered halide double perovskites, where for instance B is a 4+ metal and B' is left vacant. ${ }^{20,21}$ The most prominent examples of vacancy-ordered double perovskites are members of the $\mathrm{A}_{2} \mathrm{SnX}_{6}$ family, where $\mathrm{Sn}$ is in the $4+$ oxidation state. ${ }^{22,23}$ Double perovskites do not allow for continuous band gap tuningthe ratio between $\mathrm{B}$ and $\mathrm{B}^{\prime}$ is fixed at $1: 1$.

The band gap can be tuned by mixing two different $2+$ cations on the B-site, but reports of two fully miscible B-site cations in halide perovskites are limited. Lead and tin can be fully mixed in any 
concentration on the B-site in halide perovskites, and crystals of this type of material can be grown in solution. Lead and tin halide perovskites have similar band gaps, which limits the overall amount of band gap tunability that perovskites containing lead-tin solid solutions can achieve..$^{9,24,25}$

Other reports have found limited uptake of homovalent B-site cations (i.e., with a $2+$ charge) other than tin into a lead halide perovskite, resulting in only weak band gap tuning. ${ }^{26-31}$ It was found that when the precursor solutions have a B-site dopant concentration of less than 1 mol percent, then the dopant B-site cation will be incorporated into the parent lead halide perovskite. At higher doping concentrations, however, a secondary phase containing a higher concentration of dopant is formed at the surface. ${ }^{31}$ Existing solutionbased methods can therefore not be used to widely tune the bandgap of halide perovskites. Heterovalent Bsite doping (incorporating B-site cations of a different charge) charge is difficult, and reports of such doping are controversial because incorporating B-site cations that do not have a $2+$ charge often results in compensating defect formation elsewhere in the perovskite. ${ }^{32-34}$

Here we show, using solid-state synthesis, that the band gap of the halide perovskite $\mathrm{CsPbBr}_{3}$ can be continuously tuned over hundreds of meV through homovalent B-site doping with strontium. We synthesize the perovskite $\mathrm{CsPb}_{1-\mathrm{x}} \mathrm{Sr}_{\mathrm{x}} \mathrm{Br}_{3}(0 \leq \mathrm{x} \leq 1)$ solid solution, where $\mathrm{Sr}^{2+}$ and $\mathrm{Pb}^{2+}$ are interchangeable and randomly distributed on the $\mathrm{B}$-site. $\mathrm{Pb}^{2+}$ and $\mathrm{Sr}^{2+}$ have nearly identical ionic radii. ${ }^{35} \mathrm{CsPbBr}_{3}$ and $\mathrm{CsSrBr}_{3}$ are both known. $\mathrm{CsSrBr}_{3}$ is a wide-band gap white orthorhombic halide perovskite that is used as a scintillator when doped with rare earth ions. ${ }^{36,37}$ Compared to the 2.29(2) eV band gap of pure $\mathrm{CsPbBr}_{3}$, replacing $75 \%$ of the $\mathrm{Pb}^{2+}$ cations with $\mathrm{Sr}^{2+}$ increases the band gap by $350 \mathrm{meV}$ to 2.64(3) eV. Pure phase $\mathrm{CsSrBr}_{3}$ has a band gap beyond the visible regime, so it is colorless because it does not significantly absorb visible light. Density functional theory calculations support the observation that replacing $\mathrm{Pb}^{2+}$ with $\mathrm{Sr}^{2+}$ increases the band gap, consistent with the relative electronegativities of $\mathrm{Sr}^{2+}$ and $\mathrm{Pb}^{2+}$. These results demonstrate that homovalent B-site alloying is a viable strategy to continuously tune the band gap of halide perovskites, which may enable optical applications where it is necessary to dial in the precise band gap of a material. ${ }^{36}$ 
Figure 1 shows the powder X-ray diffraction patterns for $\mathrm{CsPbBr}_{3}$ and $\mathrm{CsSrBr}_{3}$. All the observed reflections index to reported orthorhombic unit cells. ${ }^{38,39}$ The reported lattice constants of $\mathrm{CsPbBr}_{3}$ and $\mathrm{CsSrBr}_{3}$ differ from one another by less than $1 \%$, consistent with the nearly identical ionic radii of sixcoordinate $\mathrm{Pb}^{2+}$ and $\mathrm{Sr}^{2+}$, which only differ by $0.01 \AA$ in Shannon's table. ${ }^{35}$ The powder diffraction patterns for $\mathrm{CsPb}_{1-\mathrm{x}} \mathrm{Sr}_{\mathrm{x}} \mathrm{Br}_{3}$ are shown in Figure 1, and like pure-phase $\mathrm{CsPbBr}_{3}$ and $\mathrm{CsSrBr}_{3}$ they are all orthorhombic perovskites with no obvious differences in their patterns compared to the patterns of pure-phase $\mathrm{CsPbBr}_{3}$ and $\mathrm{CsSrBr}_{3}$. Importantly, the mixed $\mathrm{Pb}-\mathrm{Sr}$ species do not exhibit any superlattice reflections when compared to $\mathrm{CsPbBr}_{3}$ and $\mathrm{CsSrBr}_{3}$, indicating that $\mathrm{Pb}^{2+}$ and $\mathrm{Sr}^{2+}$ do not order within the perovskite structure even for a 1:1 mixture of $\mathrm{Sr}$ and $\mathrm{Br}\left(\mathrm{CsPb}_{0.5} \mathrm{Sr}_{0.5} \mathrm{Br}_{3}, x=0.5\right)$. Instead, $\mathrm{Pb}^{2+}$ and $\mathrm{Sr}^{2+}$ form a solid solution where they are randomly distributed among B-sites, like what was found in mixed lead-tin halide perovskites. ${ }^{24}$ Because $\mathrm{Sr}^{2+}$ and $\mathrm{Pb}^{2+}$ have the same charge and are approximately the same size, any driving force toward ordering would have to involve the difference in their relative electronegativites, which is apparently not sufficient. In contrast, other reported mixed cation halide perovskites such as $\mathrm{Cs}_{2} \mathrm{AgBiBr}_{3}$ form double perovskites, where $\mathrm{Ag}^{+}$and $\mathrm{In}^{3+}$ are ordered within the crystal and occupy adjacent Bsites. ${ }^{11,18,40}$ Thus, B site order in heterovalent double perovskite halides appears to be promoted by the distinct charges of each of the two cations. 


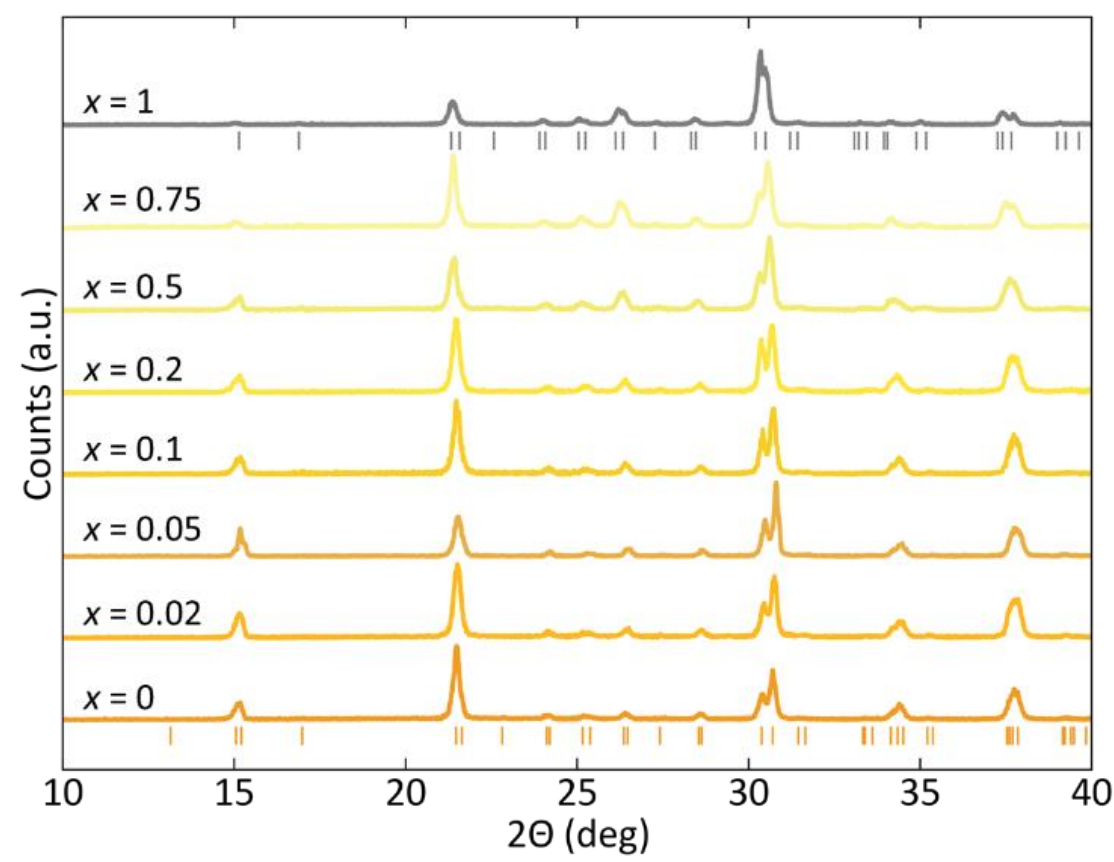

Figure 1: Structural characterization. Powder X-ray diffraction patterns of $\mathrm{CsPb}_{1-\mathrm{x}} \mathrm{Sr}_{\mathrm{x}} \mathrm{Br}_{3}(x=0-1)$. Locations of reflections for $\mathrm{CsPbr}_{3}$ and $\mathrm{CsSrBr}_{3}$ from previously reported patterns are indicated by orange and grey bars, respectively.

Figure 2a shows ground powders as well as intact pieces of $\mathrm{CsPb}_{1-\mathrm{x}} \mathrm{Sr}_{\mathrm{x}} \mathrm{Br}_{3}(x=0,0.02,0.05,0.1,0.2$, 0.5, 0.75 and 1) inside a nitrogen-filled glove box. Pseudoabsorbance spectra are shown in Figure 2b, with extracted band gaps in Figure 2c. $\mathrm{CsPBr}_{3}$ is a bright orange solid with a band gap of 2.29(2) eV, matching what has been previously reported. ${ }^{15}$ As the concentration of $\mathrm{Sr}^{2+}$ increases, the color of the material becomes a lighter orange and then a light yellow, reaching a band gap of 2.64(3) eV for $x=0.75$, which is $350 \mathrm{meV}$ larger than that of pure-phase $\mathrm{CsPbBr}_{3}$. Pure-phase $\mathrm{CsSrBr}_{3}$ is a wide band gap semiconductor that is white in color, consistent with its absorption spectrum which suggests it has an optical band gap exceeding $3.5 \mathrm{eV}$ (Figure 2b). $\mathrm{CsSrBr}_{3}$ has been explored as a scintillator for $\mathrm{X}$-ray detection when doped with 2+ rare earth cations such as $\mathrm{Eu}^{2+} .36,37$ 

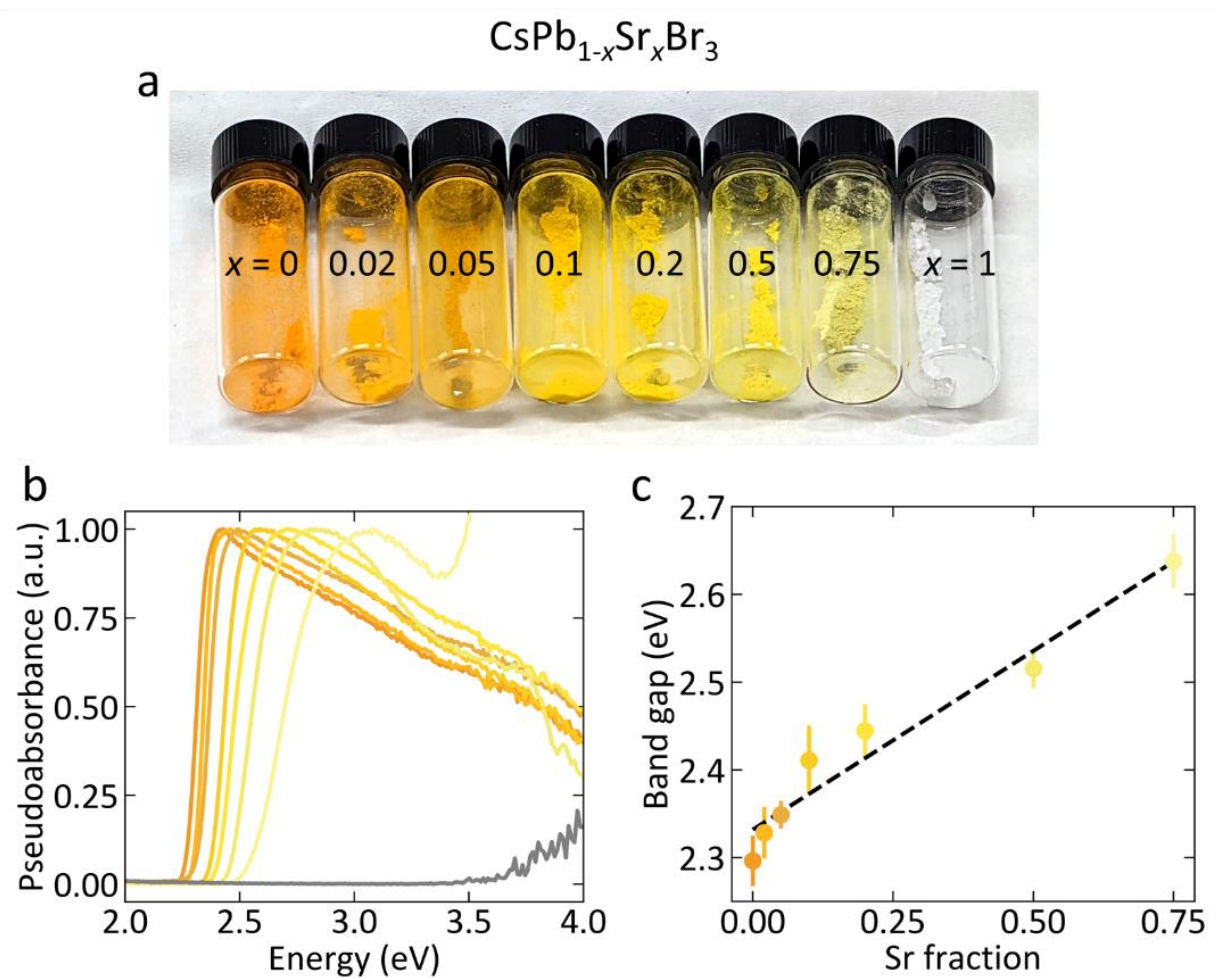

Figure 2: Optical characterization. (a) Photograph of ground powders and intact pieces of $\mathrm{CsPb}_{1-\mathrm{x}} \mathrm{Sr}_{\mathrm{x}} \mathrm{Br}_{3}$ with $x$ indicated on corresponding vial. (b) Pseudoabsorption spectra and (c) extracted band gaps for $\mathrm{CsSr}_{\mathrm{x}} \mathrm{Pb}_{1-\mathrm{x}} \mathrm{Br}_{3}$ with a linear regression $\left(R^{2}=0.95\right)$ shown in black. The color of each trace in (b) and point in (c) is set to the color of the material in (a), with pure $\mathrm{CsSrBr}_{3}$ shown in grey.

It was previously reported that the band gap of the orange-colored halide perovskite $\mathrm{CsPbBr}_{3}$ could be continuously reduced through heterovalent doping. In that case, a small amount of $\mathrm{Bi}^{3+}$ was said to replace $\mathrm{Pb}^{2+}$. Crystals incorporating $\mathrm{Bi}^{3+}$ became much darker than those of pure $\mathrm{Cs} \mathrm{PbBr}_{3} .{ }^{32}$ However, later studies showed that the intrinsic band gap was not changed through $\mathrm{Bi}^{3+}$ incorporation and instead compensating defects were formed in the crystal to offset the excess charge of the $\mathrm{Bi}^{3+}$ cation. This process creates localized states that absorb a small fraction of sub band-gap light. ${ }^{33,34}$ The increased band gap reported here from $\mathrm{Sr}^{2+}$ incorporation caused by the formation of localized defects. Mid-gap defects can only decrease the apparent band gap of the material, whereas the band gap of $\mathrm{CsPb}_{1-\mathrm{x}} \mathrm{Sr}_{\mathrm{x}} \mathrm{Br}_{3}$ increases with $\mathrm{Sr}^{2+}$ content. The bulk electronic structure of the perovskite is therefore changed through strontium incorporation. 


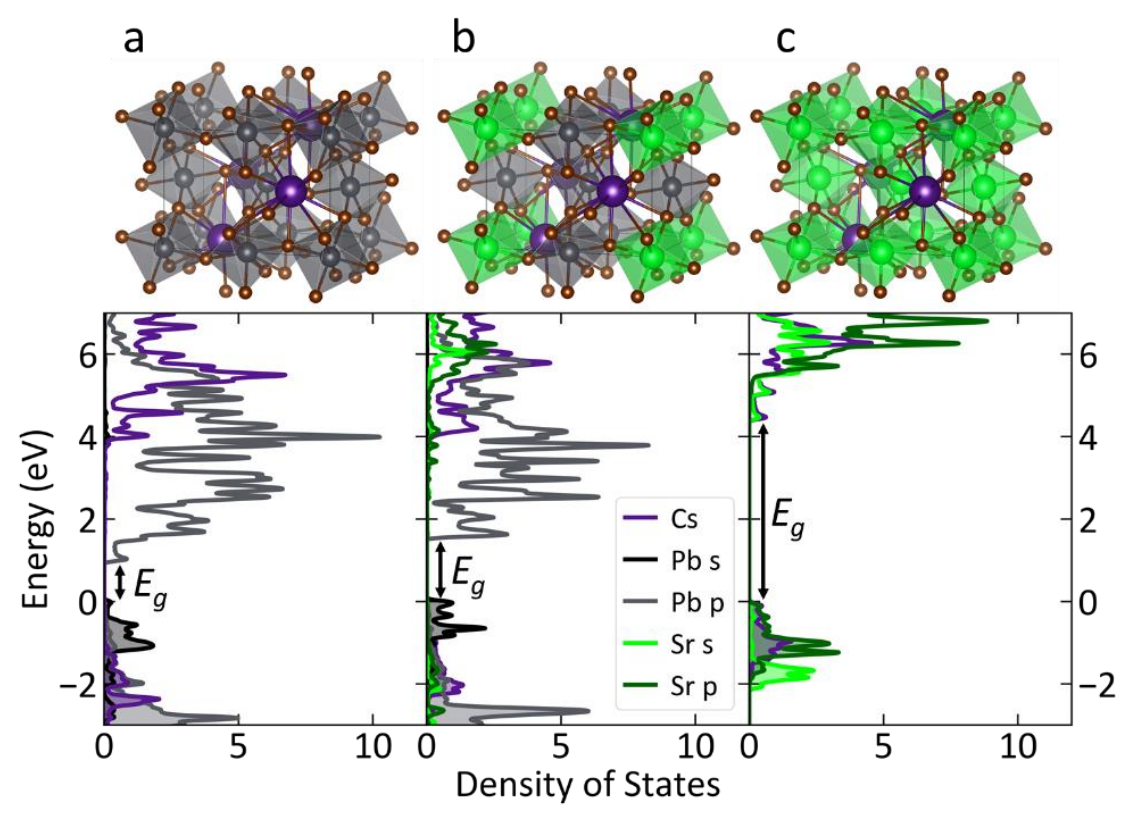

Figure 3: Results of the electronic structure calculations. Relaxed structures used to compute projected density of states (top), with $\mathrm{Cs}$ depicted in purple, $\mathrm{Pb}$ in grey, $\mathrm{Sr}$ in green, and $\mathrm{Br}$ in brown, and computed projected density of states (bottom) for (a) $\mathrm{CsPbBr}_{3}$, (b) $\mathrm{CsSr}_{0.25} \mathrm{~Pb}_{0.75} \mathrm{Br}_{3}$, and (c) $\mathrm{CsSrBr}_{3}$, with (black arrow) band gap $E_{g}$. The Fermi energy set to $0 \mathrm{eV}$ in all panels.

Density-functional theory calculations support our observation that $\mathrm{Sr}^{2+}$ substitution for $\mathrm{Pb}^{2+}$ in $\mathrm{CsPbBr}_{3}$ increases the band gap. Figure 3 a shows the projected density of states of the $\mathrm{Cs}$ and $\mathrm{Pb}$ atoms in $\mathrm{CsPbBr}_{3}$. In line with previous computations for $\mathrm{CsPbBr}_{3}$ and other lead halide perovskites, ${ }^{41,42}$ the top of the valence band is predominantly composed of halide $p$ orbitals with some contribution from $\mathrm{Pb}$ orbitals, and the bottom of the conduction band is mostly $\mathrm{Pb} p$ in character. $\mathrm{CsPbr}_{3}$ has a computed band gap of $1.00 \mathrm{eV}$, which is much smaller than the experimental value. DFT calculations are known to dramatically underestimate the band gap of lead halide perovskites, ${ }^{43}$ and our computed band gap is consistent with earlier calculations that like our calculations account for spin-orbit coupling. ${ }^{44}$

Replacing the $\mathrm{Pb}$ atom at $(0,0,0)$ with $\mathrm{Sr}$ results in the model compound $\mathrm{CsSr}_{0.25} \mathrm{~Pb}_{0.75} \mathrm{Br}_{3}$, and the projected density of states of the $\mathrm{Cs}, \mathrm{Pb}$, and $\mathrm{Sr}$ atoms is shown in Figure $3 \mathrm{~b}$. $\mathrm{CsSr}_{0.25} \mathrm{~Pb}_{0.75} \mathrm{Br}_{3}$ has a computed band gap of $1.53 \mathrm{eV}, 0.53 \mathrm{eV}$ larger than that of $\mathrm{CsPBr}_{3}$. Like in pure $\mathrm{CsPbBr}_{3}$, the top of the valence band is predominantly composed of halide $p$ orbitals with some contribution from $\mathrm{Pb} s$ orbitals, and the bottom of the conduction band is predominantly $\mathrm{Pb} p$ in character. There is negligible contribution 
of $\mathrm{Sr}$ orbitals near the top of the valence band or the bottom of the conduction band, but the presence of $\mathrm{Sr}$ in the material opens the band gap. Sr incorporation does not introduce any mid-gap states. This computational result is consistent with an earlier result that showed the computed band gap of the hybrid perovskite methylammonium lead iodide increased with $25 \% \mathrm{Ba}^{2+}$ substitution for $\mathrm{Pb}^{2+} .{ }^{27} \mathrm{We}$ note that the structural model that we used for the computation of the electronic structure of $\mathrm{CsSr}_{0.25} \mathrm{~Pb}_{0.75} \mathrm{Br}_{3}$ contains a $\mathrm{Pb}-\mathrm{Sr}$ superlattice because the $\mathrm{Sr}$ atom is located at a single well-defined crystallographic position. More sophisticated random site occupancy models for the doped material may be of interest in future calculations. Figure 3c shows the projected density of states of the $\mathrm{Cs}$ and $\mathrm{Sr}$ atoms in $\mathrm{CsSrBr}$, which has a much larger computed band gap of $4.45 \mathrm{eV}$ (Figure 3c), consistent with its wider experimental band gap.

Upon exposure to atmosphere, $\mathrm{CsPb}_{1-\mathrm{x}} \mathrm{Sr}_{\mathrm{x}} \mathrm{Br}_{3}(\mathrm{x} \neq 1)$ rapidly turns orange. Pseudoabsorbance spectra of air-exposed $\mathrm{CsPb}_{1-\mathrm{x}} \mathrm{Sr}_{\mathrm{x}} \mathrm{Br}_{3}$ are shown in Figure 4a. The band gaps of the air-exposed materials are statistically indistinguishable from that of pure $\mathrm{CsPbrr}_{3}$ (Figure 4b). Pure $\mathrm{CsSrBr}_{3}$ is deliquescent and rapidly dissolves itself when taken out of inert atmosphere. X-ray diffraction patterns of air-exposed $\mathrm{CsPb}_{1}$ ${ }_{x} \mathrm{Sr}_{x} \mathrm{Br}_{3}(\mathrm{x} \neq 1)$ are shown in Figure 4c. The diffraction patterns demonstrate that the orange color upon exposure to air is caused by the decomposition of $\mathrm{CsPb}_{1-\mathrm{x}} \mathrm{Sr}_{\mathrm{x}} \mathrm{Br}_{3}(\mathrm{x} \neq 1)$ into $\mathrm{CsPbBr}_{3}$. Two other phases are also present and are especially evident in the $x=0.5$ and 0.75 samples: $\mathrm{Cs}_{4} \mathrm{PbBr}_{6}$ (red, Figure $4 \mathrm{c}$ ) and $\mathrm{SrBr}_{2} \cdot \mathrm{H}_{2} \mathrm{O}$ (blue, Figure 4c); ${ }^{45,46}$ an additional unidentified phase containing $\mathrm{Pb}$ must also be present to account for all atoms. The hump in the absorption spectrum centered around $2.7 \mathrm{eV}$ in $\mathrm{CsPb}_{1-\mathrm{x}} \mathrm{Sr}_{\mathrm{x}} \mathrm{Br}_{3}(x=$ 0.5 and 0.75 ) is consistent with the presence of $\mathrm{Cs}_{4} \mathrm{PbBr}_{6}$, which has a band gap of $2.37 \mathrm{eV} .{ }^{47}$ There is also a strong absorption feature beginning around $3.6 \mathrm{eV}$ in the air-exposed $\mathrm{CsPb}_{1-\mathrm{x}} \mathrm{Sr}_{\mathrm{x}} \mathrm{Br}_{3}(x=0.5$ and 0.75$)$ materials, and we do not know what material causes it. Further study is needed to understand how and why exposure to air causes $\mathrm{Sr}^{2+}$ to come out of the perovskite lattice, leaving pure $\mathrm{CsPbBr}_{3}$ behind in addition to other byproducts. We hypothesize that the degradation mechanism is likely similar to the still-unknown mechanism by which metastable black perovskite-phase $\mathrm{CsPbI}_{3}$ is converted to a non-perovskite yellow phase when exposed to moisture. ${ }^{48}$ 

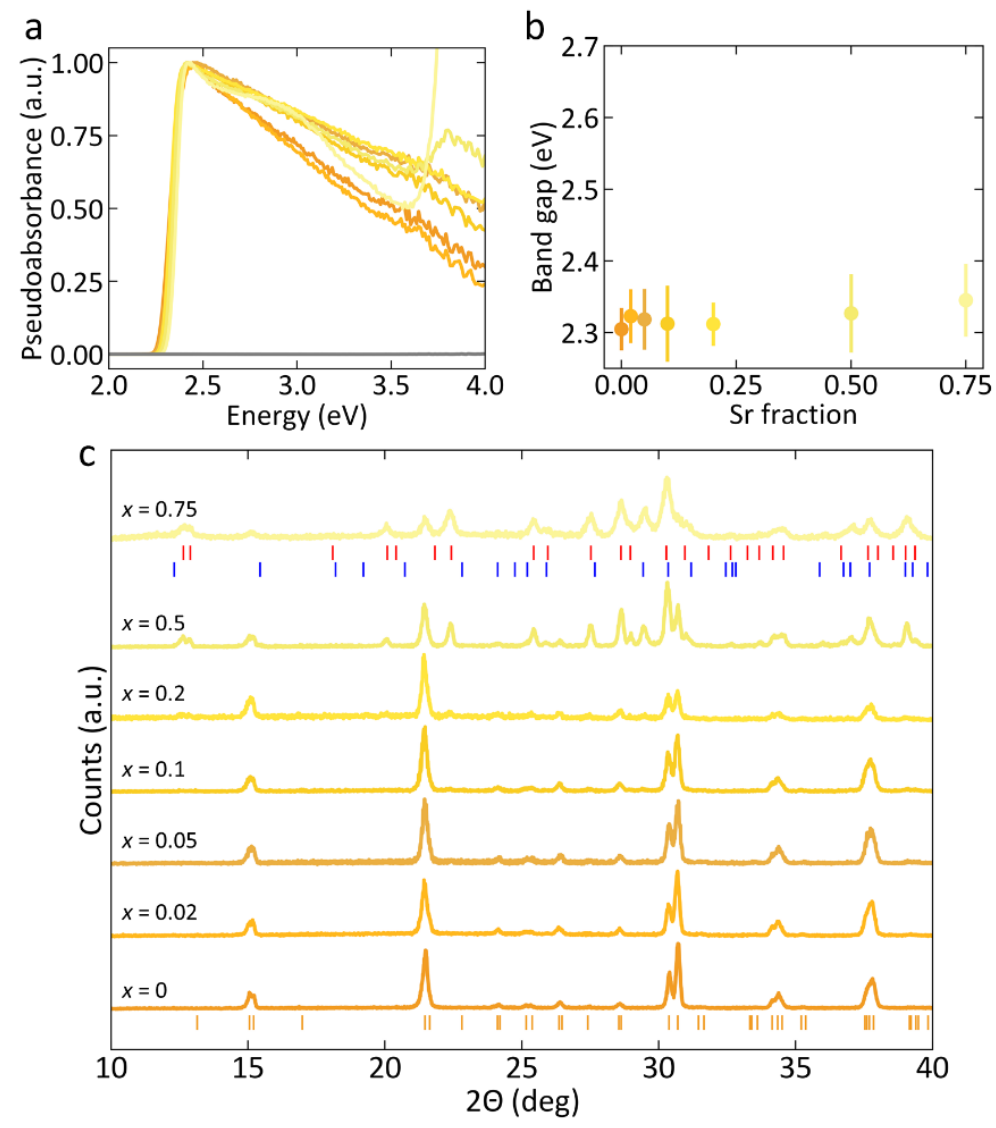

Figure 4: Sample degradation in air. (a) Pseudoabsorption spectra, (b) optical band gaps, and (c) powder $\mathrm{X}$-ray diffraction patterns of air-exposed $\mathrm{Cs} \mathrm{Pb}_{1-\mathrm{x}} \mathrm{Sr}_{\mathrm{x}} \mathrm{Br}_{3}$, with indexed reflections for $\mathrm{CsPbBr}_{3}, \mathrm{Cs}_{2} \mathrm{PbBr}_{6}$, and $\mathrm{SrBr}_{2} \cdot \mathrm{H}_{2} \mathrm{O}$ shown in orange, red, and blue, respectively.

We demonstrate that B-site alloying is a viable method to tune the band gap in halide perovskites by synthesizing and characterizing $\mathrm{CsPb}_{1-\mathrm{x}} \mathrm{Sr}_{\mathrm{x}} \mathrm{Br}_{3}(x=0-1)$. While most solution-based syntheses limit the amount of B-site dopant that can be incorporated into the perovskite lattice, solid-state synthesis removes this limitation. In $\mathrm{CsPbr}_{3}$, we replace up to $75 \%$ of $\mathrm{Pb}^{2+}$ with $\mathrm{Sr}^{2+}$, allowing the band gap to be continuously increased from 2.29(2) eV for pure $\mathrm{CsPbBr}_{3}$ to 2.64(3) eV for $\mathrm{CsPb}_{0.25} \mathrm{Sr}_{0.75} \mathrm{Br}_{3} . \mathrm{Sr}^{2+}$ and $\mathrm{Pb}^{2+}$ form a solid solution in $\mathrm{CsPb}_{1-\mathrm{x}} \mathrm{Sr}_{\mathrm{x}} \mathrm{Br}_{3}$ where they are randomly distributed throughout the lattice. The identical charge and similar size of $\mathrm{Pb}^{2+}$ and $\mathrm{Sr}^{2+}$ does not allow them to spontaneously segregate onto distinct lattice sites, unlike in charge-ordered or vacancy-ordered double perovskites. DFT calculations support the widening of the band gap of $\mathrm{CsPBr}_{3}$ upon $\mathrm{Sr}^{2+}$ substitution. In the future, it may be possible to develop solution- 
compatible processes that will allow for the ambient temperature incorporation of large amounts of homovalent B-site dopants to provide another route to tune halide perovskite band gaps for optical applications. $^{36}$

\section{Experimental}

$\mathrm{SrBr}_{2}$ (Alfa Aesar, 99\%, anhydrous) was dried overnight under rough vacuum at $230{ }^{\circ} \mathrm{C}$. It was then brought into an argon-filled glove box, loaded into a quartz tube, transferred to an ampoule sealing line, and evacuated to high vacuum. While under high vacuum, the $\mathrm{SrBr}_{2}$ was melted with a methane-oxygen torch to assist in removing residual moisture. Black impurities formed that were not soluble in molten $\mathrm{SrBr}_{2}$. The quartz tube was refilled with argon, a graphite piece was added to the top of the tube to aid in removing residual moisture, and the tube was evacuated to high vacuum $\left(10^{-4}\right.$ torr $)$ and then sealed. The ampoule was heated at $700{ }^{\circ} \mathrm{C}$ in a furnace, cooled to room temperature at $30^{\circ} \mathrm{C} / \mathrm{hr}$, and opened in an argon-filled glove box. The solidified melt containing the black impurities was loaded in a new quartz ampoule with quartz wool placed above the impure $\mathrm{SrBr}_{2}$ to act as a filter in hot centrifugation. The tube was evacuated and sealed under high vacuum, and the ampoule was then heated to $900{ }^{\circ} \mathrm{C}$ in a furnace. It was removed from the furnace while hot, inverted, and centrifuged. The quartz wool trapped the black impurities, allowing the molten $\mathrm{SrBr}_{2}$ to pass to the other end of the ampoule, where it solidified into a very light gray lump. The ampoule was opened in an argon-filled glove box, and the purified $\mathrm{SrBr} 2$ was ground into a white powder.

$\mathrm{CsPb}_{1-\mathrm{x}} \mathrm{Sr}_{\mathrm{x}} \mathrm{Br}_{3}(x=0,0.02,0.05,0.1,0.2,0.5,0.75$ and 1$)$ was synthesized by weighing out stoichiometric amounts of $\mathrm{CsBr}$ (Sigma-Aldrich, 99.999\%, anhydrous), $\mathrm{SrBr}_{2}$ (purified as described above), and $\mathrm{PbBr}_{2}$ (Alfa Aesar, 99.999\%, ultra dry) in an argon-filled glove box and placing the reagents in quartz tubes. The quartz tubes were sealed under vacuum $\left(10^{-3}\right.$ torr $)$, and the ampoules were heated to $810{ }^{\circ} \mathrm{C}$ in a furnace, melting the reagents. The ampoules were subsequently cooled to room temperature, at which point they were brought into a nitrogen-filled glove box, opened, and the melt ground in a mortar. 
Powder X-ray diffraction patterns were taken on a Rigaku Miniflex II diffractometer that was located inside a nitrogen-filled glove box, using $\mathrm{Cu} k \alpha$ radiation $(\lambda=1.5406 \AA)$. Diffuse reflectance spectra were taken on an Agilent Cary 5000 UV-Vis-NIR spectrometer equipped with an Agilent DRA-2500 internal diffuse reflectance accessory. For the diffuse reflectance measurements, $\mathrm{CsPb}_{1-\mathrm{x}} \mathrm{Sr}_{\mathrm{x}} \mathrm{Br}_{3}$ was diluted to approximately $10 \% \mathrm{w} / \mathrm{w}$ with dry $\mathrm{MgO}$ and placed in Agilent powder sample holders that were sealed with an O-ring. Diffuse reflectance spectra were converted to pseudoabsorbance spectra using the KubelkaMunk function. ${ }^{49}$ Band gaps were calculated using the direct band gap Tauc model. ${ }^{50}$

Density-functional theory calculations were performed with Quantum Espresso ${ }^{51,52}$ version 6.8 using the generalized gradient approximation functionals by Perdew, Burke, and Ernzerhof ${ }^{53}$ and PSLibrary v1.0.0 pseudopotentials. ${ }^{54}$ The starting structure of $\mathrm{CsPbr}_{3}$ employed was the crystal structure published in Ref. ${ }^{15}$ accessed through the Inorganic Crystal Structure Database ${ }^{55}$ (ICSD Collection Code 243735). The initial structure of $\mathrm{CsSrBr}_{3}$ was generated by replacing the $\mathrm{Pb}$ atoms in the $\mathrm{CsPbBr}_{3}$ structure with $\mathrm{Sr}$. The initial structure of $\mathrm{CsSr}_{0.25} \mathrm{~Pb}_{0.75} \mathrm{Br}_{3}$ was generated by converting the orthorhombic $\mathrm{CsPbBr}_{3}$ structure to triclinic $\mathrm{P}-1$ and replacing the $\mathrm{Pb}$ atom at $(0,0,0)$ with $\mathrm{Sr}$. The coordinates of all structures were optimized using the $v c$-relax routine in a scalar relativistic calculation, and the scalar relativistic-optimized structures were then optimized once more using a fully relativistic calculation. An automatically generated 5x5x5 unshifted $k$-point mesh was used for structural relaxation. The density of states was calculated using an automatically generated 10x10x10 unshifted $k$-point mesh in a fully relativistic calculation that accounts for spin-orbit coupling effects. The kinetic energy cutoff for wavefunctions was set to $60 \mathrm{Ry}$, and the kinetic energy cutoff for charge density and potential was set to 300 Ry for all calculations. The total energy convergence threshold was set to $10^{-8}$, the force convergence threshold was set to $10^{-5}$, and the selfconsistency convergence threshold was set to $10^{-8} .50 \mathrm{meV}$ of Gaussian broadening was used when generating the projected density of states.

\section{Acknowledgments}


This work is supported by the Gordon and Betty Moore Foundation as part of the EPiQS initiative under grant GBMF9066.

\section{References:}

(1) Dong, Q.; Fang, Y.; Shao, Y.; Mulligan, P.; Qiu, J.; Cao, L.; Huang, J. Electron-Hole Diffusion Lengths > 175 Um in Solution-Grown CH 3 NH 3 PbI 3 Single Crystals. Science 2015, 347, 967970.

(2) Semonin, O. E.; Elbaz, G. A.; Straus, D. B.; Hull, T. D.; Paley, D. W.; van der Zande, A. M.; Hone, J. C.; Kymissis, I.; Kagan, C. R.; Roy, X.; Owen, J. S. Limits of Carrier Diffusion in n Type and p-Type CH 3 NH 3 PbI 3 Perovskite Single Crystals. J. Phys. Chem. Lett. 2016, 7, 3510-3518.

(3) Bi, Y.; Hutter, E. M.; Fang, Y.; Dong, Q.; Huang, J.; Savenije, T. J. Charge Carrier Lifetimes Exceeding $15 \mathrm{Ms}$ in Methylammonium Lead Iodide Single Crystals. J. Phys. Chem. Lett. 2016, 7, 923-928.

(4) Zhu, X.-Y.; Podzorov, V. Charge Carriers in Hybrid-Organic-Inorganic Lead-Halide Perovskites Might Be Protected as Large Polarons. J. Phys. Chem. Lett. 2015, 6, 4758-4761.

(5) Green, M. A.; Ho-Baillie, A.; Snaith, H. J. The Emergence of Perovskite Solar Cells. Nat. Photonics 2014, 8, 506-514.

(6) NREL. Best Research-Cell Efficiencies http://www.nrel.gov/pv/assets/images/efficiency-chart.png (accessed Jan 27, 2022).

(7) Filip, M. R.; Giustino, F. The Geometric Blueprint of Perovskites. Proc. Natl. Acad. Sci. 2018, $115,5397-5402$.

(8) Goldschmidt, V. M. Die Gesetze Der Krystallochemie. Naturwissenschaften 1926, 14, 477-485.

(9) Jena, A. K.; Kulkarni, A.; Miyasaka, T. Halide Perovskite Photovoltaics: Background, Status, and Future Prospects. Chem. Rev. 2019, 119, 3036-3103.

(10) Hoffmann, R. How Chemistry and Physics Meet in the Solid State. Angew. Chemie Int. Ed. English 1987, 26, 846-878.

(11) Slavney, A. H.; Connor, B. A.; Leppert, L.; Karunadasa, H. I. A Pencil-and-Paper Method for Elucidating Halide Double Perovskite Band Structures. Chem. Sci. 2019, 10, 11041-11053.

(12) Adjokatse, S.; Fang, H. H.; Loi, M. A. Broadly Tunable Metal Halide Perovskites for Solid-State Light-Emission Applications. Mater. Today 2017, 20, 413-424.

(13) Hoke, E. T.; Slotcavage, D. J.; Dohner, E. R.; Bowring, A. R.; Karunadasa, H. I.; McGehee, M. D. Reversible Photo-Induced Trap Formation in Mixed-Halide Hybrid Perovskites for Photovoltaics. Chem. Sci. 2015, 6, 613-617. 
(14) McMeekin, D. P.; Sadoughi, G.; Rehman, W.; Eperon, G. E.; Saliba, M.; Hörantner, M. T.; Haghighirad, A.; Sakai, N.; Korte, L.; Rech, B.; Johnston, M. B.; Herz, L. M.; Snaith, H. J. A Mixed-Cation Lead Mixed-Halide Perovskite Absorber for Tandem Solar Cells. Science 2016, $351,151-155$.

(15) Linaburg, M. R.; McClure, E. T.; Majher, J. D.; Woodward, P. M. Cs1-XRbxPbCl3 and Cs1$\mathrm{XRbxPbBr} 3$ Solid Solutions: Understanding Octahedral Tilting in Lead Halide Perovskites. Chem. Mater. 2017, 29, 3507-3514.

(16) Straus, D. B.; Guo, S.; Abeykoon, A. M.; Cava, R. J. Understanding the Instability of the Halide Perovskite $\mathrm{CsPbI} 3$ through Temperature-Dependent Structural Analysis. Adv. Mater. 2020, 32, 2001069.

(17) Anderson, M. T.; Greenwood, K. B.; Taylor, G. A.; Poeppelmeier, K. R. B-Cation Arrangements in Double Perovskites. Prog. Solid State Chem. 1993, 22, 197-233.

(18) Slavney, A. H.; Hu, T.; Lindenberg, A. M.; Karunadasa, H. I. A Bismuth-Halide Double Perovskite with Long Carrier Recombination Lifetime for Photovoltaic Applications. J. Am. Chem. Soc. 2016, 138, 2138-2141.

(19) Slavney, A. H.; Leppert, L.; Saldivar Valdes, A.; Bartesaghi, D.; Savenije, T. J.; Neaton, J. B.; Karunadasa, H. I. Small-Band-Gap Halide Double Perovskites. Angew. Chemie - Int. Ed. 2018, 57, $12765-12770$.

(20) Maughan, A. E.; Ganose, A. M.; Scanlon, D. O.; Neilson, J. R. Perspectives and Design Principles of Vacancy-Ordered Double Perovskite Halide Semiconductors. Chem. Mater. 2019, 31, 11841195.

(21) Brik, M. G.; Kityk, I. V. Modeling of Lattice Constant and Their Relations with Ionic Radii and Electronegativity of Constituting Ions of A2XY6 Cubic Crystals (A=K, Cs, Rb, Tl; X=tetravalent Cation, Y=F, Cl, Br, I). J. Phys. Chem. Solids 2011, 72, 1256-1260.

(22) Lee, B.; Stoumpos, C. C.; Zhou, N.; Hao, F.; Malliakas, C.; Yeh, C. Y.; Marks, T. J.; Kanatzidis, M. G.; Chang, R. P. H. Air-Stable Molecular Semiconducting Iodosalts for Solar Cell Applications: Cs2SnI6 as a Hole Conductor. J. Am. Chem. Soc. 2014, 136, 15379-15385.

(23) Maughan, A. E.; Ganose, A. M.; Candia, A. M.; Granger, J. T.; Scanlon, D. O.; Neilson, J. R. Anharmonicity and Octahedral Tilting in Hybrid Vacancy-Ordered Double Perovskites. Chem. Mater. 2018, 30, 472-483.

(24) Hao, F.; Stoumpos, C. C.; Chang, R. P. H.; Kanatzidis, M. G. Anomalous Band Gap Behavior in Mixed $\mathrm{Sn}$ and $\mathrm{Pb}$ Perovskites Enables Broadening of Absorption Spectrum in Solar Cells. J. Am. Chem. Soc. 2014, 136, 8094-8099.

(25) Zhao, B.; Abdi-Jalebi, M.; Tabachnyk, M.; Glass, H.; Kamboj, V. S.; Nie, W.; Pearson, A. J.; Puttisong, Y.; Gödel, K. C.; Beere, H. E.; Ritchie, D. A.; Mohite, A. D.; Dutton, S. E.; Friend, R. H.; Sadhanala, A. High Open-Circuit Voltages in Tin-Rich Low-Bandgap Perovskite-Based Planar Heterojunction Photovoltaics. Adv. Mater. 2017, 29.

(26) Pérez-del-Rey, D.; Forgács, D.; Hutter, E. M.; Savenije, T. J.; Nordlund, D.; Schulz, P.; Berry, J. J.; Sessolo, M.; Bolink, H. J. Strontium Insertion in Methylammonium Lead Iodide: Long Charge 
Carrier Lifetime and High Fill-Factor Solar Cells. Adv. Mater. 2016, 28, 9839-9845.

(27) Zhang, H.; Shang, M. hui; Zheng, X.; Zeng, Z.; Chen, R.; Zhang, Y.; Zhang, J.; Zhu, Y. Ba2+ Doped $\mathrm{CH} 3 \mathrm{NH} 3 \mathrm{PbI} 3$ to Tune the Energy State and Improve the Performance of Perovskite Solar Cells. Electrochim. Acta 2017, 254, 165-171.

(28) Mondal, A.; Lata, A.; Gupta, S. Effect of Sr2+ Doping on Optical, Thermal and Photocatalytic Behaviour of CsPbBr3. Mater. Lett. 2021, No. November, 131314.

(29) Karunakaran, S. K.; Arumugam, G. M.; Yang, W.; Ge, S.; Khan, S. N.; Mai, Y.; Lin, X.; Yang, G. Europium (II)-Doped All-Inorganic CsPbBr3 Perovskite Solar Cells with Carbon Electrodes. Sol. RRL 2020, 2000390, 1-8.

(30) Zhao, Y.; Wang, Y.; Duan, J.; Yang, X.; Tang, Q. Divalent Hard Lewis Acid Doped CsPbBr 3 Films for 9.63\%-Efficiency and Ultra-Stable All-Inorganic Perovskite Solar Cells. J. Mater. Chem. A 2019, 7, 6877-6882.

(31) Phung, N.; Félix, R.; Meggiolaro, D.; Al-Ashouri, A.; Sousa e Silva, G.; Hartmann, C.; Hidalgo, J.; Köbler, H.; Mosconi, E.; Lai, B.; Gunder, R.; Li, M.; Wang, K.-L.; Wang, Z.-K.; Nie, K.; Handick, E.; Wilks, R. G.; Marquez, J. A.; Rech, B.; Unold, T.; Correa-Baena, J.-P.; Albrecht, S.; De Angelis, F.; Bär, M.; Abate, A. The Doping Mechanism of Halide Perovskite Unveiled by Alkaline Earth Metals. J. Am. Chem. Soc. 2020, 142, 2364-2374.

(32) Abdelhady, A. L.; Saidaminov, M. I.; Murali, B.; Adinolfi, V.; Voznyy, O.; Katsiev, K.; Alarousu, E.; Comin, R.; Dursun, I.; Sinatra, L.; Sargent, E. H.; Mohammed, O. F.; Bakr, O. M. Heterovalent Dopant Incorporation for Bandgap and Type Engineering of Perovskite Crystals. J. Phys. Chem. Lett. 2016, 7, 295-301.

(33) Nayak, P. K.; Sendner, M.; Wenger, B.; Wang, Z.; Sharma, K.; Ramadan, A. J.; Lovrinčić, R.; Pucci, A.; Madhu, P. K.; Snaith, H. J. Impact of Bi3+ Heterovalent Doping in Organic-Inorganic Metal Halide Perovskite Crystals. J. Am. Chem. Soc. 2018, 140, 574-577.

(34) Lozhkina, O. A.; Murashkina, A. A.; Shilovskikh, V. V.; Kapitonov, Y. V.; Ryabchuk, V. K.; Emeline, A. V.; Miyasaka, T. Invalidity of Band-Gap Engineering Concept for Bi3+ Heterovalent Doping in CsPbBr3 Halide Perovskite. J. Phys. Chem. Lett. 2018, 9, 5408-5411.

(35) Shannon, R. D. Revised Effective Ionic Radii and Systematic Studies of Interatomic Distances in Halides and Chalcogenides. Acta Crystallogr. Sect. A 1976, 32, 751-767.

(36) Gokhale, S. S.; Stand, L.; Lindsey, A.; Koschan, M.; Zhuravleva, M.; Melcher, C. L. Improvement in the Optical Quality and Energy Resolution of CsSrBr3: Eu Scintillator Crystals. J. Cryst. Growth 2016, 445, 1-8.

(37) Suta, M.; Larsen, P.; Lavoie-Cardinal, F.; Wickleder, C. Photoluminescence of CsMBr3:Eu2+ $(\mathrm{M}=\mathrm{Mg}, \mathrm{Ca}, \mathrm{Sr})$ - A Novel Strategy for the Development of Low-Energy Emitting Phosphors. $J$. Lumin. 2014, 149, 35-44.

(38) Rodová, M.; Brožek, J.; Knížek, K.; Nitsch, K. Phase Transitions in Ternary Caesium Lead Bromide. J. Therm. Anal. Calorim. 2003, 71, 667-673.

(39) Meyer, G.; Schilling, G. CsSrBr3 PDF 00-044-1375. ICDD Grant-in-Aid. Inst. Anorganische 
Chemie, Univ. Hannover: Hannover, Germany 1993.

(40) McClure, E. T.; Ball, M. R.; Windl, W.; Woodward, P. M. Cs 2 AgBiX 6 (X = Br, Cl): New Visible Light Absorbing, Lead-Free Halide Perovskite Semiconductors. Chem. Mater. 2016, 28, 1348-1354.

(41) Yin, W. J.; Shi, T.; Yan, Y. Unique Properties of Halide Perovskites as Possible Origins of the Superior Solar Cell Performance. Adv. Mater. 2014, 26, 4653-4658.

(42) Manser, J. S.; Christians, J. A.; Kamat, P. V. Intriguing Optoelectronic Properties of Metal Halide Perovskites. Chem. Rev. 2016, 116, 12956-13008.

(43) Perdew, J. P. Density Functional Theory and the Band Gap Problem. Int. J. Quantum Chem. 2009, 28, 497-523.

(44) Ghaithan, H. M.; Alahmed, Z. A.; Qaid, S. M. H.; Hezam, M.; Aldwayyan, A. S. Density Functional Study of Cubic, Tetragonal, and Orthorhombic CsPbBr 3 Perovskite. ACS Omega 2020, 5, 7468-7480.

(45) Engelen, B.; Freiburg, C.; Lutz, H. D. Zur Kenntnis Der Hydrate Des Typs MX 2 - 1 H 2 O Mit M $=\mathrm{Sr}, \mathrm{Ba}$ Und $\mathrm{X}=\mathrm{Cl}, \mathrm{Br}$, I. Kristallstrukturen Des Strontiumchlorid-Monohydrats, $\mathrm{SrCl} 2 \cdot 1 \mathrm{H} 2$ O, Und Des Strontiumbromid-Monohydrats, $\mathrm{SrBr} 2 \cdot 1 \mathrm{H} 2$ O. Zeitschrift für Anorg. und Allg. Chemie 1983, 497, 151-156.

(46) Velázquez, M.; Ferrier, A.; Péchev, S.; Gravereau, P.; Chaminade, J.-P.; Portier, X.; Moncorgé, R. Growth and Characterization of Pure and Pr3+-Doped Cs4PbBr6 Crystals. J. Cryst. Growth 2008, $310,5458-5463$.

(47) Andrews, R. H.; Clark, S. J.; Donaldson, J. D.; Dewan, J. C.; Silver, J. Solid-State Properties of Materials of the Type Cs4MX6(Where $\mathrm{M}=\mathrm{Sn}$ or $\mathrm{Pb}$ and $\mathrm{X}=\mathrm{Cl}$ or Br). J. Chem. Soc. Dalt. Trans. 1983, No. 4, 767.

(48) Straus, D. B.; Guo, S.; Cava, R. J. Kinetically Stable Single Crystals of Perovskite-Phase CsPbI 3. J. Am. Chem. Soc. 2019, 141, 11435-11439.

(49) Hecht, H. G. The Interpretation of Diffuse Reflectance Spectra. J. Res. Natl. Bur. Stand. Sect. A Phys. Chem. 1976, 80A, 567.

(50) Viezbicke, B. D.; Patel, S.; Davis, B. E.; Birnie, D. P. Evaluation of the Tauc Method for Optical Absorption Edge Determination: ZnO Thin Films as a Model System. Phys. status solidi 2015, 252, 1700-1710.

(51) Giannozzi, P.; Baroni, S.; Bonini, N.; Calandra, M.; Car, R.; Cavazzoni, C.; Ceresoli, D.; Chiarotti, G. L.; Cococcioni, M.; Dabo, I.; Dal Corso, A.; de Gironcoli, S.; Fabris, S.; Fratesi, G.; Gebauer, R.; Gerstmann, U.; Gougoussis, C.; Kokalj, A.; Lazzeri, M.; Martin-Samos, L.; Marzari, N.; Mauri, F.; Mazzarello, R.; Paolini, S.; Pasquarello, A.; Paulatto, L.; Sbraccia, C.; Scandolo, S.; Sclauzero, G.; Seitsonen, A. P.; Smogunov, A.; Umari, P.; Wentzcovitch, R. M. QUANTUM ESPRESSO: A Modular and Open-Source Software Project for Quantum Simulations of Materials. J. Phys. Condens. Matter 2009, 21, 395502.

(52) Giannozzi, P.; Andreussi, O.; Brumme, T.; Bunau, O.; Nardelli, M. B.; Calandra, M.; Car, R.; 
ChemRxiv

Cavazzoni, C.; Ceresoli, D.; Cococcioni, M.; others. Advanced Capabilities for Materials Modelling with Quantum ESPRESSO. (ArXiv:1709.10010v1 [Cond-Mat.Mtrl-Sci]). J. Phys. Condens. Matter 2017, 29, 465901.

(53) Perdew, J. P.; Burke, K.; Ernzerhof, M. Generalized Gradient Approximation Made Simple. Phys. Rev. Lett. 1996, 77, 3865-3868.

(54) Dal Corso, A. Pseudopotentials Periodic Table: From H to Pu. Comput. Mater. Sci. 2014, 95, 337350 .

(55) Hellenbrandt, M. The Inorganic Crystal Structure Database (ICSD) - Present and Future. In Crystallography Reviews; 2004; Vol. 10, pp 17-22. 